\title{
Case - Acquired hemophilia $A$ in a patient with metastatic castration-resistant prostate cancer
}

Michael Poscente; Chun Loo Gan; Daniel Y.C. Heng; Nimira Alimohamed

${ }^{1}$ Tom Baker Cancer Centre, University of Calgary, Calgary, AB, Canada

Cite as: Poscente M, Gan CL, Heng DYC, et al. Case - Acquired hemophilia A in a patient with metastatic castration-resistant prostate cancer. Can Urol Assoc J 2021 February 12; Epub ahead of print. http://dx.doi.org/10.5489/cuaj.7089

Published online February 12, 2021

$* * *$

\section{Introduction}

Hematologic abnormalities in patients with non-hematologic malignancies are relatively common with nearly $10 \%$ experiencing abnormal bleeding ${ }^{1,2}$. The etiology ranges from bone marrow suppression to more acutely life-threatening conditions such as DIC or trauma to highly vascularized neoplastic tissue ${ }^{3}$. Acquired hemophilia A (AHA) is a rare condition with an incidence of 1.5 in 1000000 annually $^{4}$ and is associated with the development of autoantibodies to clotting factor $\mathrm{VIII}^{2}$. AHA can be caused by autoimmune disease, drug exposure, post-partum, and malignancies ${ }^{5}$.

\section{Case report}

A 62-year-old man with metastatic castrate-resistant prostate cancer (mCRPC) presented to emergency medical attention with a two-day history of swelling and bruising in the right hand, in the context of four months of spontaneous bruising and a recent non-traumatic psoas muscle hematoma. His medical profile was significant for prostate cancer. Initial prostate biopsy four years prior to this presentation revealed intermediate-risk prostate adenocarcinoma with Gleason $4+3=7$ disease and a pretreatment PSA of $12.5 \mathrm{ug} / \mathrm{L}$. He was treated with external beam radiotherapy and short-term androgen deprivation therapy (ADT) in the form of leuprolide acetate given subcutaneously (Figure 1). Post-treatment toxicity included intermittent rectal bleeding. Biochemical recurrence of his prostate cancer occurred 17 months later, and the patient was restarted on ADT, subsequently given continuously. He developed metastatic castrateresistant disease and was commenced on abiraterone and prednisone 30 months after his initial diagnosis, with biopsy-proven lymphadenopathy. Eventually the patient developed bone metastases and he was switched to second line mCRPC therapy with docetaxel chemotherapy after being on abiraterone and prednisone for 16 months. Docetaxel was commenced at 75\% dose for tolerability one month prior to his emergency room presentation. 
The patient's presenting concern in the emergency department was significant bruising in his right hand (Figure 2A). Two days prior to presentation he noted opening a jar, which caused swelling, pain, and decreased range of motion. There was no epistaxis, hematemesis, melena, headaches or focal neurological symptoms, and the small amount of rectal bleeding was unchanged. He did not have clinical features consistent with compartment syndrome. He noted gross hematuria three weeks prior which had spontaneously resolved, with ongoing blood-tinged urine. There was no history of personal or familial hematologic disorders. The patient had been finding it easier to bruise since January of 2020, with progression of severity. He was found to have an intramuscular psoas hematoma related to a ground level fall in May of 2020 which was conservatively managed.

His medications included ASA $81 \mathrm{mg}$ PO for a remote vertebral artery stroke, prednisone $5 \mathrm{mg}$ PO BID started with the docetaxel, alendronate $70 \mathrm{mg}$ weekly, hydromorphone PRN, pantoprazole $40 \mathrm{mg}$ PO, rosuvastatin $5 \mathrm{mg}$ QHS, calcium and vitamin D supplements. Vital signs were stable. Initial blood work revealed low hemoglobin of $63 \mathrm{~g} / \mathrm{L}$ [137-180g/L], normal INR of 1.2, elevated PTT of 63.4 seconds [28-38s], elevated fibrinogen of $4.2 \mathrm{~g} / \mathrm{L}$ [1.6-4.1g/L], and elevated D-dimer at $4.85 \mathrm{mg} / \mathrm{L}[<0.5 \mathrm{mg} / \mathrm{L}]$. DVT of the upper extremity was ruled out with ultrasound.

The patient was admitted to hospital for further work up and management, requiring red blood cell transfusions and tranexamic acid. Initially the patient was managed with Factor VII 7 mg IV TID (90 mcg/kg) ${ }^{6}$ with Factor VIII (FVIII) 7200 IU IV BID while awaiting hemostasis studies. Two days later, inhibitor to factor 8 was confirmed at a level of $11 \mathrm{BU} / \mathrm{ml}$ (Figure 3 ). FVIII 3500 IU IV BID was given for 4 days. The patient was given prednisone $70 \mathrm{mg}$ PO daily ( $1 \mathrm{mg} / \mathrm{kg}$ ) for 11 days, $60 \mathrm{mg}$ PO daily for 20 days, and $50 \mathrm{mg}$ PO daily for 14 days. Rituximab $710 \mathrm{mg}$ ( $\left.375 \mathrm{mg} / \mathrm{m}^{\wedge} 2\right)$ was started one week after presentation due to a lack of improvement on steroids and was repeated q7days for 3 times.

As the perfusion of his hand improved the bruising extended up the arm (Figure 2B). The range of motion in his hand improved as swelling decreased. He developed further episodes of lower extremity hematoma after early physiotherapy, however these improved as his clotting factors normalized and he returned to his baseline mobility. To treat his underlying malignancy, enzalutamide $160 \mathrm{mg}$ was started once the patient was clinically stable and was continued as an outpatient. At discharge after 3 months of hospital stay the patient was biochemically and physiologically stable on a prednisone taper, PJP prophylaxis, enzalutamide $160 \mathrm{mg}$ daily, tamsulosin $0.4 \mathrm{mg}$ daily, and his other home medications. The patient ultimately did not respond to enzalutamide and was started on cabazitaxel chemotherapy with good clinical and PSA response; his factor 8 levels normalized [0.5-1.5U/ml] and his inhibitor levels are nearly undetectable (Figure 3). 


\section{Discussion}

Acquired Hemophilia A (AHA) is a rare and serious disease caused by the development of autoantibodies against factor VIII. Due to its rarity, the true incidence of acquired hemophilia is unknown. The best estimate of the incidence of AHA comes from a series of 172 patients in a two-year national surveillance study by the United Kingdom Hemophilia Centre Doctors' organization, where an incidence of $1.48 \mathrm{cases} / \mathrm{million} / \mathrm{year}$ were reported ${ }^{4}$. In this series, underlying malignancy accounted for $15 \%$ of the cases, of which $2 \%$ (3 patients) had prostate cancer. In another series of 41 patients with AHA, 6 patients were found to have underlying prostate cancer ${ }^{7}$.

There are numerous causes of abnormal bleeding in the cancer patient, and this case was complicated by long term steroid use, aspirin ${ }^{8}$, and recent commencement of chemotherapy which initially was considered as a potential cause of the development of AHA. As in this case, patients with AHA often present with spontaneous mucosal bleeding, hematomas, or hematuria, and contrary to inherited hemophilia, hemarthrosis is rare ${ }^{9}$. Presentation may be related to surgery or interventions ${ }^{10}$, and rarely may represent initial presentation of malignancy ${ }^{11}$. In this case, the patient's progressive disease may have triggered the development of AHA. Most patients have a high response to immunosuppression, with a median time to remission of 5 weeks $^{12}$. The mortality of AHA ranges from $17-22 \%$ of cases $^{13}$. Survival outcomes are related to FVIII levels, while higher inhibitor concentration, age $>74$, male gender, and associated malignancy have been shown to be negative prognostic factors ${ }^{14}$.

The goal of treatment of AHA is to achieve hemostasis and eradicate antibodies ${ }^{15}$. Eradication is achieved with immunosuppressive therapy, with the most common therapy being cyclophosphamide and steroids ${ }^{12}$. In this case rituximab with steroids was chosen instead given his recent chemotherapy and the concern of added toxicities from further chemotherapy ${ }^{16}$. If possible, treating the underlying malignancy eradicates FVIII antibodies faster ${ }^{7}$, and so this patient was started on enzalutamide in hospital and cabazitaxel in the community for treatment of progressive metastatic castrate-resistant disease. Given the mortality associated with complications from immunosuppressive therapy ${ }^{14}$, prophylactic antibiotics and monitoring for metabolic/cardiovascular derangements continued as an outpatient. In this case the patient survived and has no further hematologic concerns.

\section{Conclusions}

AHA is an uncommon complication of malignancy. Because of its rarity, a high index of suspicion and prompt recognition of potential bleeding diathesis in a cancer patient is crucial to the investigation and management of this life-threatening condition. The involvement of a multidisciplinary team with members from the oncology, hematology and blood bank is critical in the management of this highly lethal condition. Immunosuppression and treatment of the 
underlying malignancy appears to play a central role in management, as demonstrated in our case.

\section{References}

1. Reeves, B. N. \& Key, N. S. Acquired hemophilia in malignancy. Thrombosis Research 2012;129:S66-68.

2. Lewis, A., Joseph, J., Pathak, N., et al. Acquired factor VIII deficiency in prostate adenocarcinoma presenting as multiple hematomas and hemarthrosis. SAGE Open Med Case Rep 2020;8.

3. Escobar, M. A. Bleeding in the patient with a malignancy: is it an acquired factor VIII inhibitor? Cancer 2012;118:312-20.

4. Collins, P. W., Hirsch, S., Baglin, T., et al. Acquired hemophilia A in the United Kingdom: a 2-year national surveillance study by the United Kingdom Haemophilia Centre Doctors' Organisation. Blood 2007;109:1870-77.

5. Alidoost, M., Conte, G. A., Chaudry, R., et al. A Unique presentation of spontaneous compartment syndrome due to acquired hemophilia A and associated malignancy: case report and literature review. World Journal of Oncology 2020;11:72-5.

6. Franchini, M. \& Lippi, G. Acquired factor VIII inhibitors. Blood 2008;112:250-55.

7. Sallah, S. \& Wan, J. Y. Inhibitors against factor VIII in patients with cancer. Analysis of 41 patients. Cancer 2001;91:1067-74.

8. Okiro, J. O., Khan, A. Z., Keane, F., et al. Aspirin unmasking acquired haemophilia A in a patient with prostate cancer. BMJ Case Rep 2016;2016:bcr2016216890.

9. Coppola, A., Favaloro, E., Tufano, A., et al. Acquired inhibitors of coagulation factors: part I-acquired hemophilia A. Seminars in Thrombosis and Hemostasis 2012;38:433-46.

10. Celestino, F., Verri, C., De Carlo, F., et al. Localised prostate cancer and hemophilia A (AHA): Case report and management of the disease. Arch Ital Urol Androl 2014;86:22728.

11. Sheth, C., Gill, A. \& Sekhon, S. Life-threatening hemorrhage from acquired hemophilia A as a presenting manifestation of prostate cancer. $J$ Community Hosp Intern Med Perspect 2016;6:32461.

12. Tiede, A., Collins, P., Knoebl, P., et al. International recommendations on the diagnosis and treatment of acquired hemophilia A. Haematologica 2020;105:1791-801.

13. Godaert, L., Bartholet., S., Colas, S., et al. Acquired hemophilia A in aged people: A systematic review of case reports and case series. Seminars in Hematology 2018;55:197201.

14. Tiede, A., Klamroth, R., Scharg, R., et al. Prognostic factors for remission of and survival in acquired hemophilia A (AHA): results from the GTH-AH 01/2010 study. Blood 2015;125:1091-97.

15. Charlebois, J., Rivard, G.-É. \& St-Louis, J. Management of acquired hemophilia A: Review of current evidence. Transfusion and Apheresis Science 2018;57:717-20.

16. Franchini, M. Rituximab in the treatment of adult acquired hemophilia A: a systematic review. Crit. Rev. Oncol. Hematol. 2007;63:47-52. 


\section{Figures and Tables}

Fig. 1. Timeline of disease diagnosis, management, and progression. Serial prostate-specific antigen (PSA) measurements are presented in graph form.
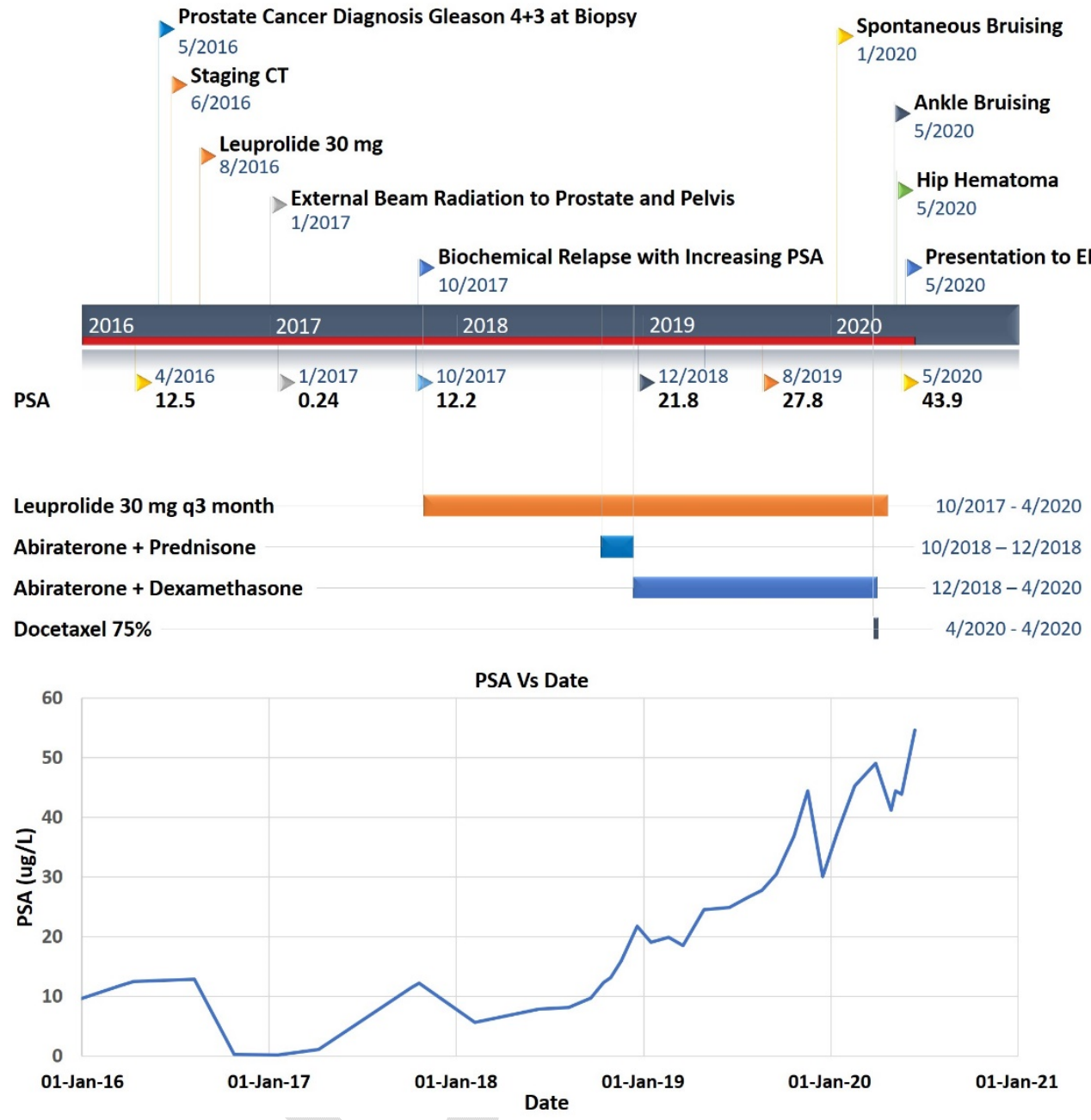
Fig. 2. (A) Initial bruising in the hand. (B) Progression of the bruising 4 days later.

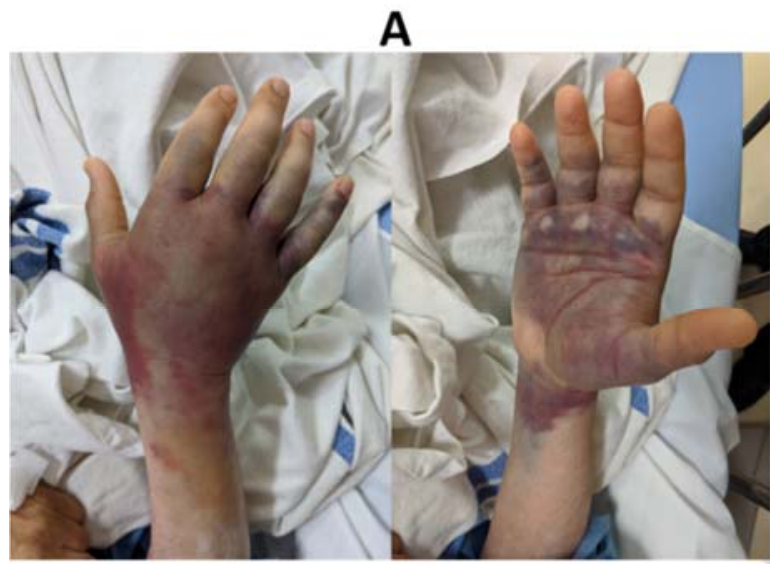

Bruising at Presentation

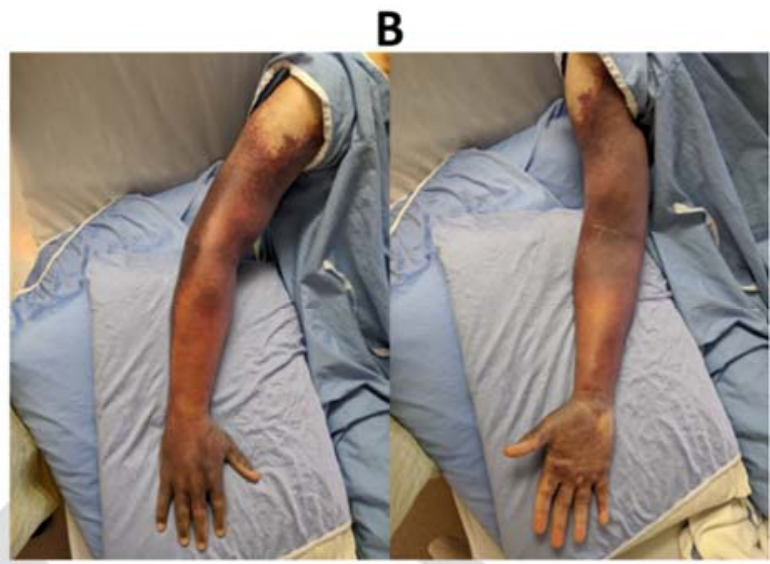

Bruising Four Days After Presentation

Fig. 3. Factor 8 and factor 8 inhibitor levels after diagnosis. Reference ranges for Factor 8 activity provided with dotted orange lines. In the case of the FVIII inhibitor, any activity is considered abnormal

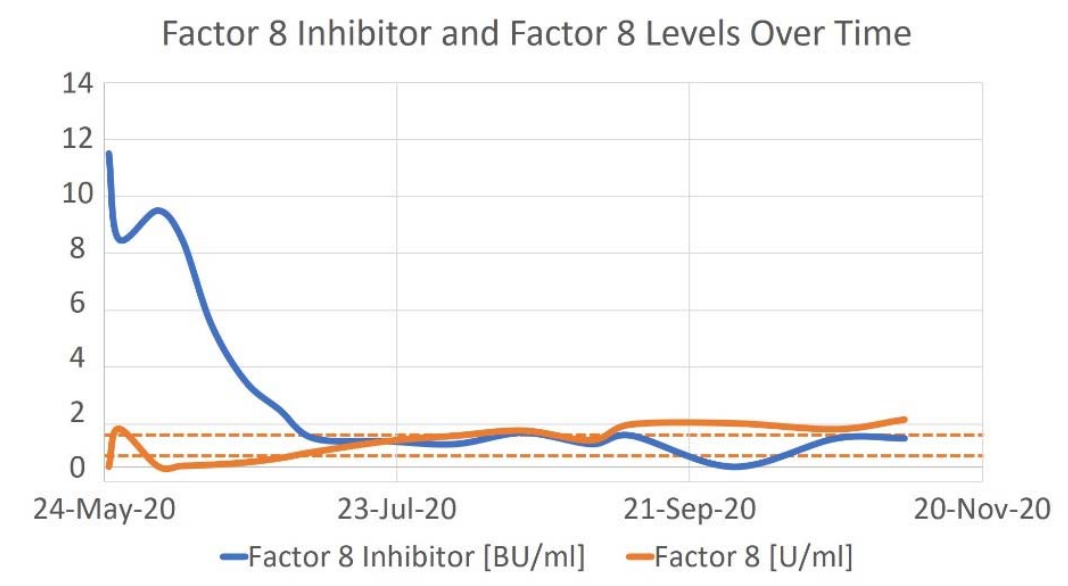

